\title{
The genome and transcriptome of Japanese flounder provide insights into flatfish asymmetry
}

\author{
Changwei Shao ${ }^{1,2,14}$, Baolong Bao ${ }^{3,14}$, Zhiyuan Xie, ${ }^{4,14}$, Xinye Chen ${ }^{3,14}$, Bo Li ${ }^{4,14}$, Xiaodong Jia ${ }^{1,2,14}$, Qiulin Yao ${ }^{4}$, \\ Guillermo Ortí ${ }^{5}$, Wenhui $\mathrm{Li}^{4}$, Xihong $\mathrm{Li}^{1,2}$, Kristin Hamre ${ }^{6,7}$, Juan $\mathrm{Xu}^{3}$, Lei Wang ${ }^{1,2}$, Fangyuan Chen ${ }^{4}$, \\ Yongsheng Tian ${ }^{1,2}$, Alex M Schreiber ${ }^{8}$, Na Wang ${ }^{1,2}$, Fen Wei ${ }^{3}$, Jilin Zhang ${ }^{4}$, Zhongdian Dong ${ }^{1,2}$, Lei Gao ${ }^{3}$, \\ Junwei Gai ${ }^{3}$, Takashi Sakamoto ${ }^{9}$, Sudong Mo ${ }^{1,2}$, Wenjun Chen ${ }^{3}$, Qiong Shi ${ }^{4}$, Hui Li ${ }^{3}$, Yunji Xiu ${ }^{1,2}$, Yangzhen $\mathrm{Li}^{1,2}$, \\ Wenteng Xu ${ }^{1,2}$, Zhiyi Shi ${ }^{3}$, Guojie Zhang ${ }^{4}$, Deborah M Power ${ }^{10,11,15}$, Qingyin Wang ${ }^{1,2,15}$, Manfred Schartl ${ }^{12,13,15}$ \\ \& Songlin Chen ${ }^{1,2,15}$
}

Flatfish have the most extreme asymmetric body morphology of vertebrates. During metamorphosis, one eye migrates to the contralateral side of the skull, and this migration is accompanied by extensive craniofacial transformations and simultaneous development of lopsided body pigmentation ${ }^{1-5}$. The evolution of this developmental and physiological innovation remains enigmatic. Comparative genomics of two flatfish and transcriptomic analyses during metamorphosis point to a role for thyroid hormone and retinoic acid signaling, as well as phototransduction pathways. We demonstrate that retinoic acid is critical in establishing asymmetric pigmentation and, via cross-talk with thyroid hormones, in modulating eye migration. The unexpected expression of the visual opsins from the phototransduction pathway in the skin translates illumination differences and generates retinoic acid gradients that underlie the generation of asymmetry. Identifying the genetic underpinning of this unique developmental process answers long-standing questions about the evolutionary origin of asymmetry, but it also provides insight into the mechanisms that control body shape in vertebrates.

To study the role of genomic architecture in asymmetry development during flatfish metamorphosis (Fig. 1a), we produced a high-quality reference genome $(546 \mathrm{Mb})$ of the Japanese flounder (Paralichthys olivaceus). We generated $52.6 \mathrm{~Gb}$ of effective Illumina sequencing data, enabling high coverage ( 120 -fold) to support contig and scaffold N50 sizes of $30.5 \mathrm{~kb}$ and $3.9 \mathrm{Mb}$, respectively (Supplementary
Figs. 1 and 2, Supplementary Tables 1 and 2, and Supplementary Note). Almost all of the assembled fragments (98\% of scaffolds) were anchored onto 24 chromosomes on the basis of high-resolution genetic maps, accounting in total for $535 \mathrm{Mb}$ of the assembled genome $^{6,7}$ (Supplementary Fig. 3 and Supplementary Table 3). Comparison of the Japanese flounder genome to the genome of another, distantly related flatfish, the Chinese tongue sole (Cynoglossus semilaevis $)^{8}$, yielded extensive stretches of synteny (394 Mb) despite divergence around 70 Mya (Fig. 1b). These highly syntenic regions accounted for about $85 \%$ of the two genomes (Supplementary Table 4 and Supplementary Note). Transcriptome sequences (20.5 Gb) aided gene annotation (Supplementary Table 5) and identified 21,787 protein-coding genes for Japanese flounder and 15,534 gene models resolving orthology relationships between Japanese flounder and Chinese tongue sole (Supplementary Tables 6 and 7, and Supplementary Note).

To characterize the genetic mechanisms that facilitated the evolution of flatfish metamorphosis, we identified gene families present in flatfish but absent from six other teleosts. We found 153 such families that were enriched in the Gene Ontology (GO) terms "collagen," "microtubule," "regulation of appetite," and "protein polymerization," and in the Kyoto Encyclopedia of Genes and Genomes (KEGG) pathways related to cell proliferation and apoptosis, carbohydrate metabolism, and cytokines and immune responses (Supplementary Figs. 4-6, Supplementary Tables 8-10, and Supplementary Note). Within the 12 flatfish gene family expansions (Fisher's exact test, $P<0.05$ ) (Fig. 1b and Supplementary Table 11), five contained 83 known

\footnotetext{
${ }^{1}$ Yellow Sea Fisheries Research Institute, CAFS, Key Laboratory for Sustainable Development of Marine Fisheries, Ministry of Agriculture, Qingdao, China. ${ }^{2}$ Laboratory for Marine Fisheries Science and Food Production Processes, Qingdao National Laboratory for Marine Science and Technology, Qingdao, China. ${ }^{3}$ The Key Laboratory of Exploration and Utilization of Aquatic Genetic Resources, Shanghai Ocean University, Ministry of Education, Shanghai, China. ${ }^{4}$ BGI-Shenzhen, Shenzhen, China. ${ }^{5}$ Department of Biological Sciences, George Washington University, Washington, DC, USA. ${ }^{6}$ National Institute of Nutrition and Seafood Research (NIFES), Bergen, Norway. ${ }^{7}$ Department of Biology, University of Bergen, Bergen, Norway. ${ }^{8}$ St. Lawrence University, Biology Department, Canton, New York, USA. ${ }^{9}$ Graduate School of Marine Science and Technology, Tokyo University of Marine Science and Technology, Tokyo, Japan. ${ }^{10}$ Centro de Ciências do Mar (CCMAR), Comparative Endocrinology and Integrative Biology, Universidade do Algarve, Faro, Portugal. ${ }^{11}$ College of Fisheries and Life Science, Shanghai Ocean University, Ministry of Education, Shanghai, China. ${ }^{12}$ Physiologische Chemie, University of Würzburg, Biozentrum, Am Hubland, and Comprehensive Cancer Center Mainfranken, University Clinic Würzburg, Würzburg, Germany. ${ }^{13}$ Institute for Advanced Studies, Texas A\&M University, College Station, Texas, USA. ${ }^{14}$ These authors contributed equally to this work. 15These authors jointly directed this work. Correspondence should be addressed to S.C. (chensl@ysfri.ac.cn), M.S. (phch1@biozentrum.uni-wuerzburg.de), Q.W. (qywang@public.qd.sd.cn) or D.M.P. (dpower@ualg.pt).
} 
a

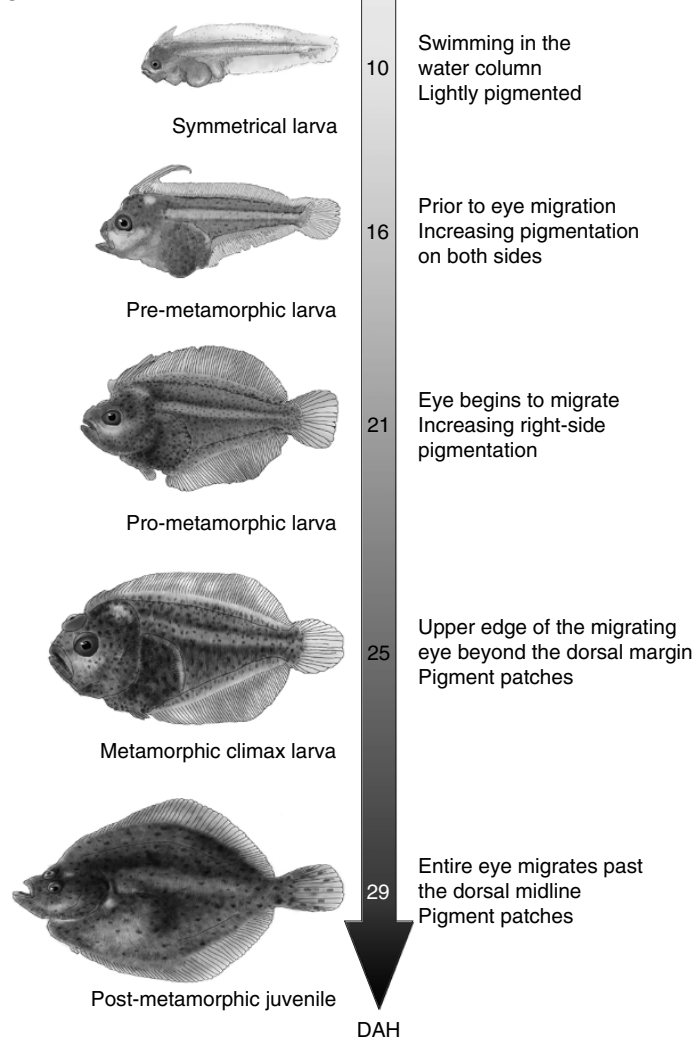

b

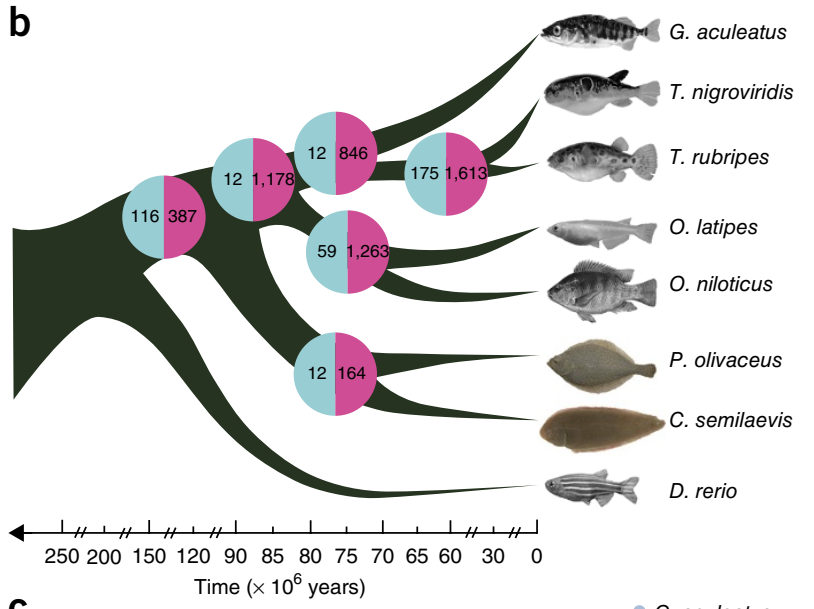

C

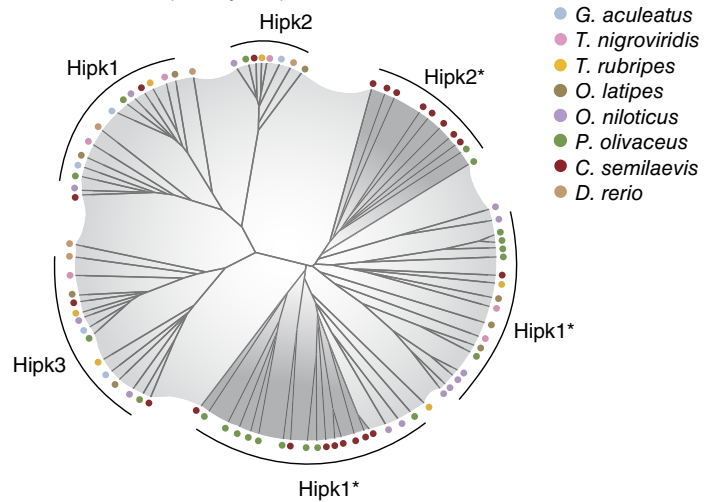

Figure 1 Flatfish metamorphosis and phylogenomic evolution. (a) Gross morphology of metamorphosis in Japanese flounder. DAH, days after hatching. (b) Maximum-likelihood tree from eight teleost species. The number of gene-family expansion events is indicated in turquoise, and that of gene-family contraction events in pink. (c) Flatfish-specific expansion of the hipk gene families. Phylogenetic analysis of deduced Hipk proteins from teleost fish generated two major clusters. One cluster (left side) comprised the three subgroups of Hipk1, Hipk2, and Hipk3, which are distinct from one another. Gene numbers in all analyzed species were very similar in the subgroups. The second cluster (right side) of Hipk proteins was enriched for flatfishlineage-specific expansions, in particular one expanded Hipk2-like (Hipk2*) clade in Chinese tongue sole and one expanded Hipk1-like (Hipk1*) clade in both Chinese tongue sole and Japanese flounder.

genes that are involved in cellular apoptosis, regulation of eye size, retinal neurotransmission, and cartilage scaffolding. Such processes are predominant among the dramatic morphological and functional modifications during flatfish metamorphosis (Supplementary Tables 11-13). This is further emphasized by the link with the expansion of the homeodomain-interacting protein kinase (Hipk) gene family, which is involved in p53-mediated cellular apoptosis and regulation of eye size, lens formation, and retinal lamination during late embryogenesis ${ }^{9}$ (Fig. 1c and Supplementary Note), as well as in the dramatic changes in the eyes during metamorphosis ${ }^{10}$.

We further identified 106 positively selected genes (PSGs) in the flatfish lineage (Supplementary Tables 14-19) that had amino acid substitutions at positions that are otherwise highly conserved in other fish taxa (Supplementary Fig. 7 and Supplementary Note). PSGs in the ancestral flatfish were genes associated with axial patterning (irx5 and usp16), neural patterning (arid1a and igsf8), musculoskeletal restructuring (bmp1, ano6, col10a1, pepd, nr1d2, mybpc1, and myo1h), thyroid hormone (TH) signaling (notch2), retinoic acid (RA) signaling (aldh1l1, aldh3b1, and $r d h 14$ ), and genes of the phototransduction pathway ( $g u c y 2 f$ and $g r k 1$ ) (Fig. 2 and Supplementary Note).

We reasoned that comparative transcriptomics of well-defined developmental stages of Japanese flounder and Chinese tongue sole (Fig. 1a and Supplementary Table 5), cross-referenced with genes identified in flatfish but absent from other teleosts or PSGs, should yield the master genes, namely, genes responsive to THs that occupy a central position in the gene networks underpinning the tissue transformations in flatfish metamorphosis. We identified 2,307 differentially expressed orthologous genes (DEGs) that were common in both species when DEGs identified by pairwise comparison of each metamorphic stage were analyzed (Supplementary Table 20). This large gene set was enriched for several pathways that can be linked to recognized morphological or physiological changes during metamorphosis (for example, TH action, photo- and olfactory transduction, and nutrition and metabolism), and it contains many genes that are involved in neural development and remodeling, anterior-posterior patterning, cell proliferation, and photoreceptor development (Supplementary Figs. 8-10, Supplementary Tables 21-27, and Supplementary Note). PSGs were represented within the DEGs in the flatfish lineage during metamorphosis (16.04\%), and the proportion identified was similar to the ratio of DEGs to non-positively selected genes in flatfish (14.84\%) (Supplementary Tables 17 and 18, and Supplementary Note).

A role for THs in flatfish metamorphosis was identified in the early $1980 \mathrm{~s}^{11}$. THs are obligatory for vertebrate metamorphosis ${ }^{12}$ and function by binding to nuclear-localized receptors (Thra and Thrb), or they may have a nongenomic cellular role and activate a complex hierarchical cascade of target genes ${ }^{13-15}$ (Fig. 3a). The essential role of THs in flatfish metamorphosis ${ }^{16}$ was reiterated in the current study by the detection of 21 differentially expressed orthologs of the 
a

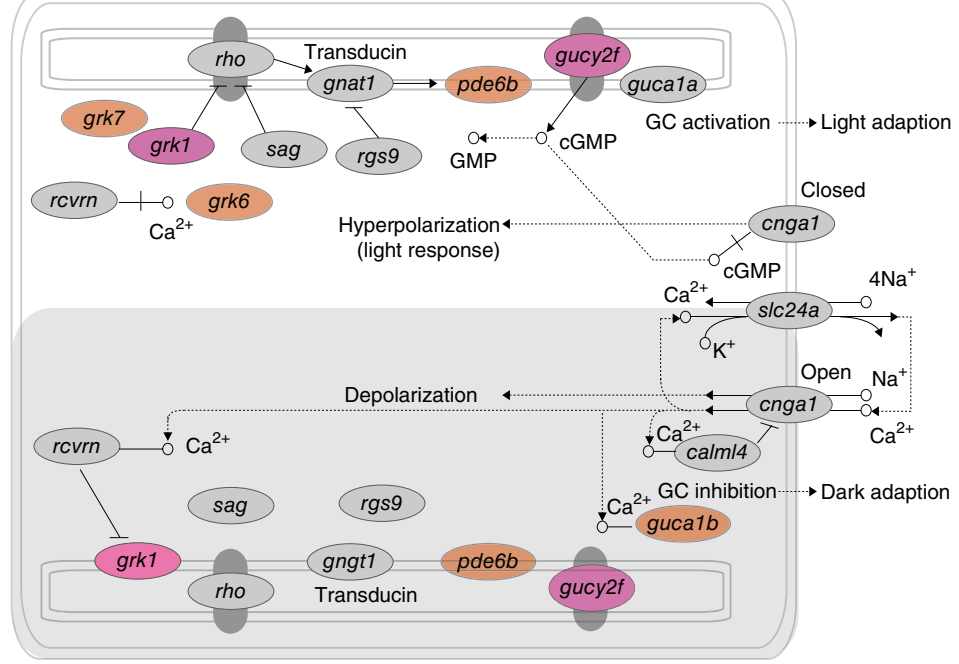

b

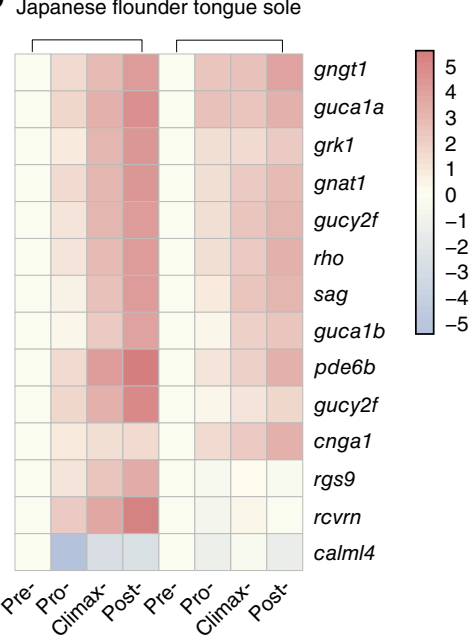

C

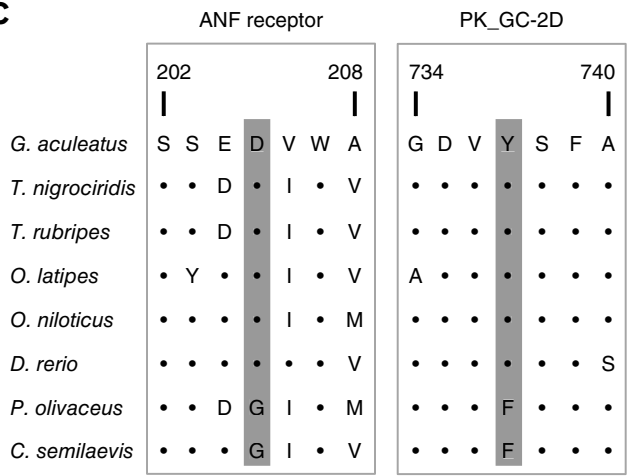

d
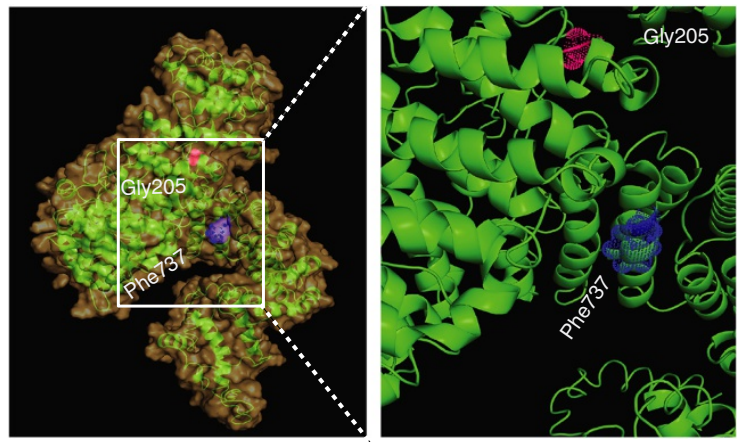

Figure 2 Visual adaptation of flatfish. (a) Positive selection of genes in the phototransduction pathway in flatfish. Genes under positive selection in the flatfish ancestor (gucy2f and grk1) are highlighted in pink. Genes under positive selection in only Japanese flounder (grk6, grk7, pde6b, and guca1b) are highlighted in orange. (b) Consistent stage-specific differential expression of gene orthologs involved in the phototransduction pathway during metamorphosis. The expression of orthologs increases gradually in the pre-metamorphic to post-metamorphic stages, with the exception of rgs9, rcvrn, and calm/4. Pre-, pre-metamorphic larva; Pro-, pro-metamorphic larva; Climax-, metamorphic climax larva; Post-, post-metamorphic larva. Three larvae were used for each developmental stage. (c) Mutations under positive selection that are unique to flatfish (gray shading) in the functional domain of the ANF receptor and the PK_GC-2D domain in Gucy2f. (d) The location of unique mutations in a three-dimensional model of Gucy2f. One positively selected site (Phe737; in blue) is located in an $\alpha$-helix, and the other site (Gly205; in pink) is on the random coil.

TH signaling pathway in Chinese tongue sole and Japanese flounder (Supplementary Table 28). Distribution or expression of thyroxine (T4), tri-iodothyronine (T3), deiodinase 2 (dio2), dio3, thraa, and thrb 1 colocalized around the eyes during Japanese flounder metamorphosis, and this pattern became progressively asymmetric with the advance toward climax (Supplementary Figs. 11-18). Treatment with methimazole (MMI), an inhibitor of TH synthesis that blocks flatfish metamorphosis ${ }^{4}$, inhibited eye migration (Supplementary Fig. 19 and Supplementary Table 29) and reduced the asymmetric expression of dio3, thraa, and thrb1 (Supplementary Figs. 20 and 21).

RAs are ligands of the nuclear receptors Rars (the ligand is alltrans RA, or ATRA) and Rxrs (the ligand is 9-cis-RA), which form heterodimers with $\mathrm{TH}$ receptors ${ }^{17}$ and are well-recognized morphogens involved in lateralization ${ }^{18}$. The strong positive selection of genes involved in the RA system in Japanese flounder (aldh1l2 and rbp4b) and Chinese tongue sole (aldh1l1 and $r d h 12$ ) (Supplementary Tables 17 and 18) and the enrichment of genes of the RA pathway in the DEGs during metamorphosis ( $r x r a, r x r g, r a r g, a l d h 1 l 2, r b p 2$, raet 1, rdh12, rbp3, and lrat) (Supplementary Table 30) suggest their involvement in asymmetry formation. We hypothesized that DEGs of the RA signaling pathway might explain the acquisition of $\mathrm{TH}$ signaling pathway asymmetry during metamorphosis, and that this cross-talk could be the basis for the generation of asymmetry in flatfish. Genes encoding isoforms of RA receptors (Rxra and Rxrb) that heterodimerize in yeast two-hybrid assays (Supplementary Figs. 22 and 23) with Thraa and Thrb1, respectively, showed marked left-right asymmetric gene expression in the epithelial tissue surrounding the eyes in the pro-metamorphic stage, when the eye starts to migrate (Supplementary Fig. 24 and Supplementary Note), thereby preceding the pronounced asymmetry that develops at the morphological level. Other genes encoding proteins involved in RA metabolism (such as cyp26, rbp2, and aldh1-3) showed variable degrees of asymmetric expression at the metamorphic climax (Supplementary Figs. 25 and 26). MMI-the inhibitor of TH synthesis that reduced the asymmetric expression of dio3, thraa, and thrb1-had the same effect on expression of rxra and rara (Supplementary Fig. 27). In summary, genes of the TH and RA signaling pathways had coincident expression patterns in the region of the head most profoundly modified during metamorphosis. Injection of 9-cis-RA or ATRA into the suborbital area of the prospective blind side eye of pre-metamorphic Japanese 
a

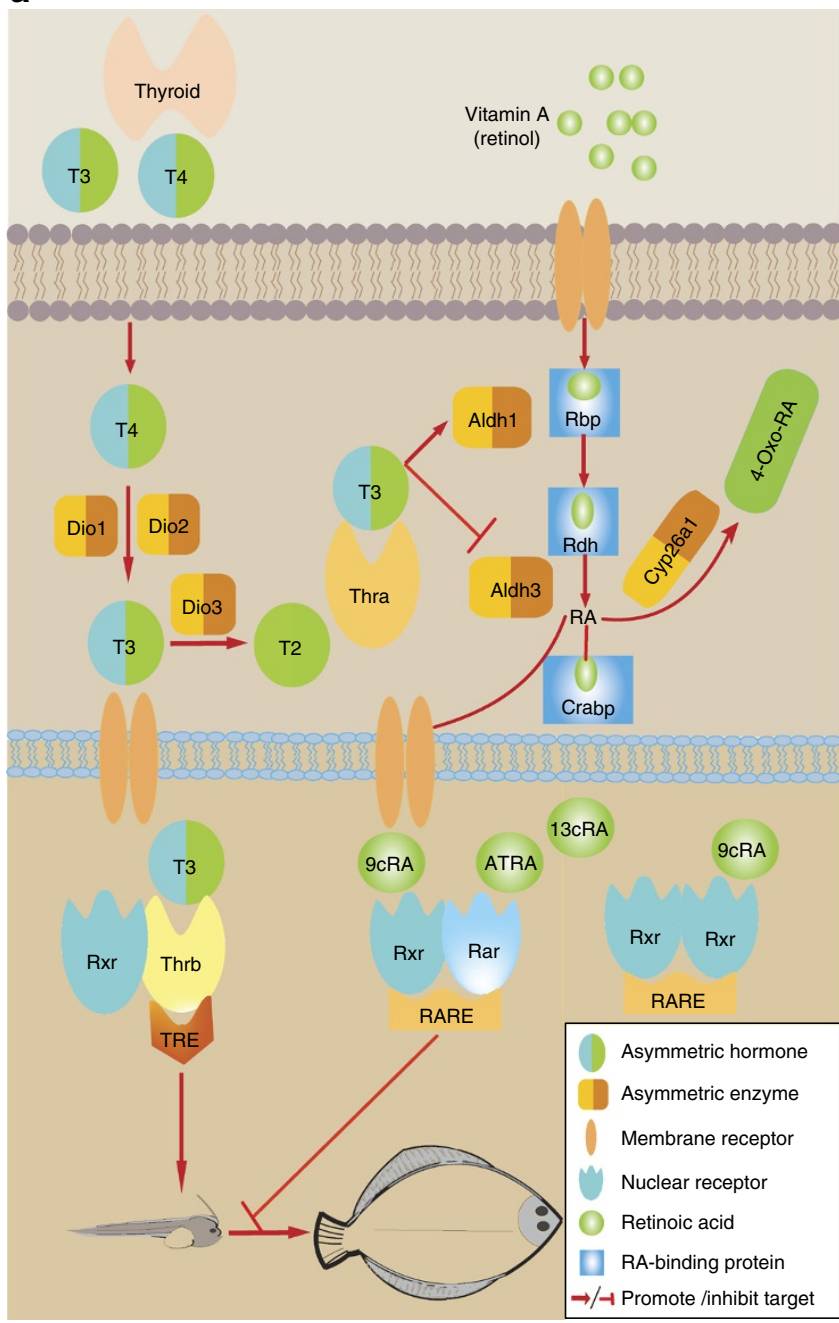

b

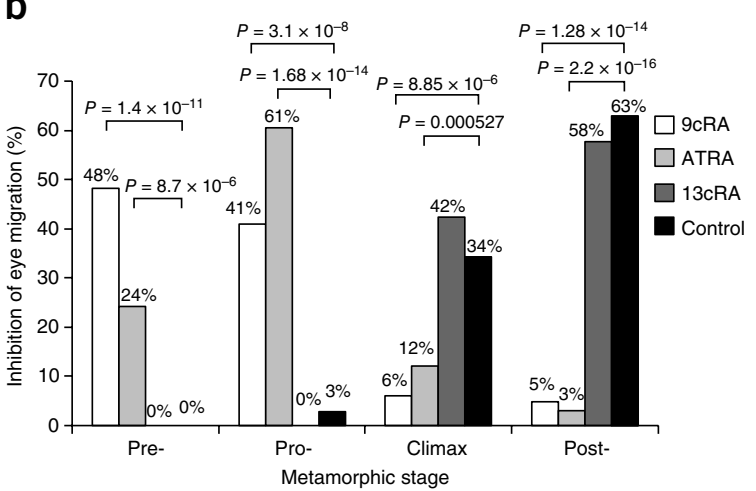

C
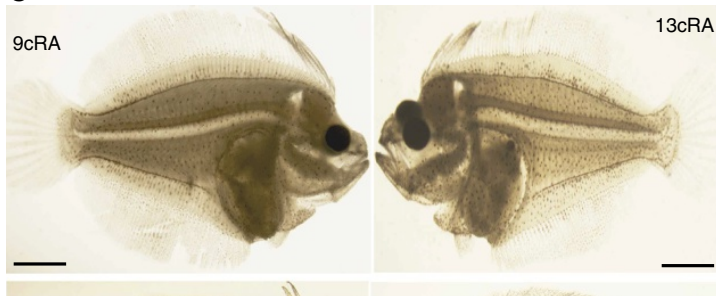

$13 \mathrm{cRA}$

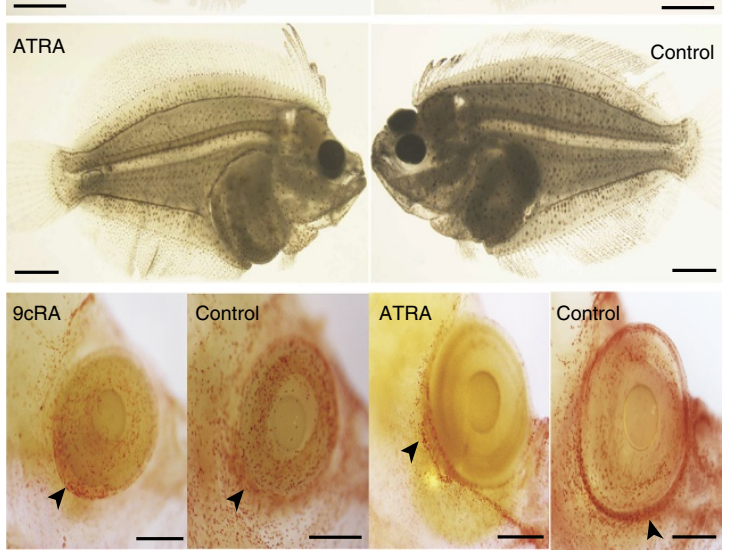

Figure 3 Molecular signatures in flatfish eye migration. (a) Putative cross-talk between TH signaling and RA signaling in eye migration. The cross-talk between RA and T3 in flatfish metamorphosis is hypothesized to consist of competition between the liganded Thrb and Rar for heterodimerization with the liganded Rxr. The heterodimers bind to TRE and RARE, respectively, to regulate gene transcription29. 9cRA, 9-cis-RA; 13cRA, 13-cis-RA.

(b) Inhibition of eye migration at $33 \mathrm{DAH}$ in Japanese flounder at different metamorphic stages after injection of different RA isoforms. DMSO was used as the control. 9-cis-RA and ATRA, which have the highest affinity for Rxrs and Rars, blocked eye migration most effectively, whereas 13-cis-RA, which has low affinity for the receptors, was less effective. $P$ values, as determined by chi-square test, indicate significant differences between the blind and ocular sides in the different treatments relative to the control. 9-cis-RA treatment, $n=83$ individuals survived; ATAR treatment, $n=99$ individuals survived; 13-cis-RA treatment, $n=59$ individuals survived; control group with DMSO treatment, $n=70$ individuals survived. (c) Representative phenotypes caused by microinjection of RAs. The images show eye symmetry observed in specimens injected with 9-cis-RA (blind side) (top left, $n=4$ ) or ATRA (blind side) (middle left, $n=3$ ); eye asymmetry was observed in specimens injected with 13-cis-RA (ocular side) (top right, $n=34$ ) or DMSO (ocular side) (control; middle right, $n=44$ ). Scale bars, $1 \mathrm{~mm}$. The bottom row of images shows cell proliferation in the suborbital area of the blind-side eye $^{30}$. The black arrowheads indicate the proliferating epidermal cells in the assay used to detect proliferation. Proliferating cells in the blind side of Japanese flounder injected with 9-cis-RA and ATRA were less numerous than in control larvae at the pro-metamorphic stage. Scale bars, $200 \mu \mathrm{m}$.

flounder larvae inhibited eye migration in 95\% (chi-square tests, $P=1.28 \times 10^{-14}$ ) and $97 \%$ (chi-square tests, $P=2.2 \times 10^{-16}$ ) of the larvae, respectively. Injection of a noncompeting Rxra ligand, 13-cis-RA, inhibited eye migration in $42 \%$ of the larvae (versus $37 \%$ for the DMSO control) (Fig. 3b and Supplementary Fig. 28). As expected, the proliferation of cells in the suborbital area of the blind side eye was notably reduced in 9-cis-RA- and ATRA-treated larvae as compared to that in the DMSO-treated controls (Fig. 3c and Supplementary Fig. 29). ATRA treatment led to upregulation of rara expression and downregulation of thraa and thrb1 expression, but it had no effect on the other retinoic acid receptors (Supplementary Figs. 30 and 31). Taken together, these results suggest that during metamorphosis, eye migration in Japanese flounder is directly regulated by the interaction of RA- and TH-induced signaling pathways.

Pigmentation also becomes asymmetric during flatfish metamorphosis, and an association exists between impaired eye migration and the failure to develop an asymmetric pigmentation pattern in skin ${ }^{19}$. We therefore tested the hypothesis that the left-right asymmetric RA gradients important for eye migration might also establish asymmetric pigmentation ${ }^{20}$. We did this by determining the concentration of RA in the pigmented (ocular) and the unpigmented (blind) skin and by comparing the two skin transcriptomes (ocular and blind) of Japanese flounder and Chinese tongue sole (Supplementary Note). The concentrations of ATRA and 9-cis-RA in the ocular side skin 
a

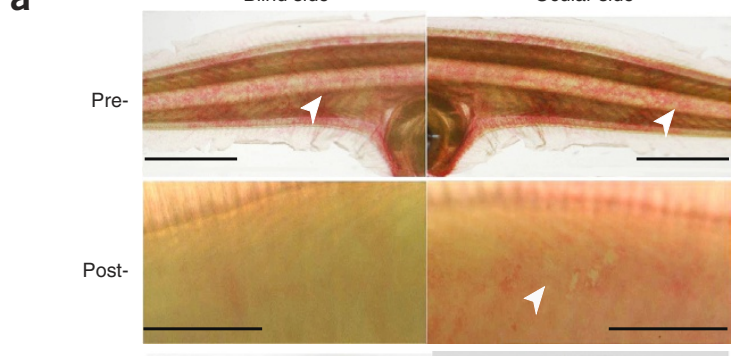

C

100 DAH

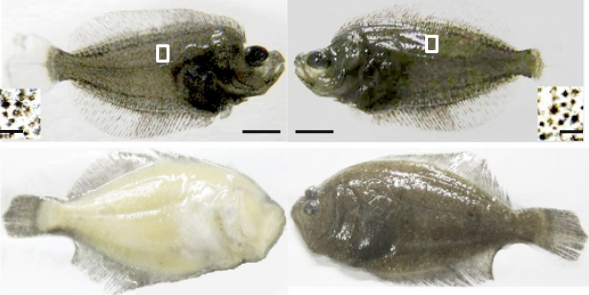

b
Ocular side

Pre-

Pro-

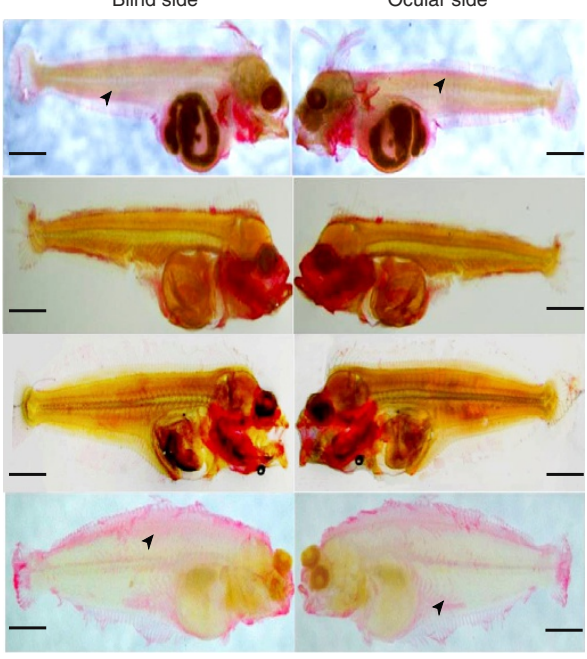

d
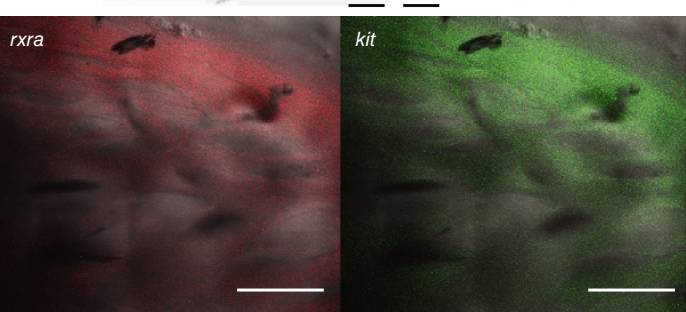

sws2

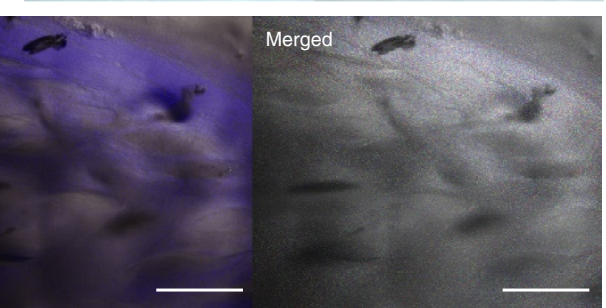

Figure 4 Visual opsins establish the asymmetric RA gradient during flatfish metamorphosis. (a) Representative images $(n=3)$ showing the asymmetric expression pattern of aldh3 in the body skin during metamorphosis in Japanese flounder. No difference in aldh3 expression was detected on either side of the pre-metamorphic larvae, but large differences existed in post-metamorphic juveniles. White arrowheads indicate positive signal. Scale bars, $1 \mathrm{~mm}$. (b) Representative images $(n=3$ ) showing the ubiquitous expression of opsin (sws2) in the skin during metamorphosis of Japanese flounder. Black arrowheads indicate positive signal. Scale bars, $0.5 \mathrm{~mm}$. (c) Adult chromatophore distribution on the blind side (left) and ocular side (right) of juveniles $100 \mathrm{~d}$ after irradiation by blue light (upper) $(n=4)$ and in unirradiated controls (below) $(n=90)$. Scale bars, $10 \mathrm{~mm}$. Insets show highermagnification views of adult pigment cells (scale bars, $0.5 \mathrm{~mm}$ ). (d) Co-expression of rxra, kit, and sws 2 in the pigment cells of skin, as detected by three-color fluorescence in situ hybridization, in fish at the post-metamorphic stage. rxra, kit, and sws2 mRNA were detected with digoxygenin (red), biotin (green), and fluorescein (blue), respectively. Scale bars, $100 \mu \mathrm{m}$. For more detailed information, see Supplementary Figures 42-50.

$(0.0396 \mathrm{ng} / \mathrm{mg}$ and $0.1430 \mathrm{ng} / \mathrm{mg}$, respectively) were substantially $(\sim 10 \times)$ and significantly higher than in the blind side skin $(0.0036 \mathrm{ng} /$ mg ATRA, one-way analysis of variance (ANOVA), $P=0.0294 ; 0.0172$ ng/mg 9-cis-RA, one-way ANOVA, $P=0.0452$ ) (Supplementary Fig. 32). Genes involved in pigment cell development and differentiation (such as mlana, tyrp1, and $d c t$, among others) (Supplementary Fig. 33) and in the pigmentation pathway (Supplementary Table 31) were upregulated in the pigmented skin. In contrast, genes involved in the RA pathway (rara, rxrb, and aldh1) and TH signaling (thrb1 and dio2) had similar abundance in pigmented and unpigmented integument (Supplementary Fig. 34), although in situ hybridization analyses indicated that rxra and aldh 3 expression were asymmetric (Fig. 4a and Supplementary Figs. 35-38). Injection of 9-cis-RA and ATRA into the dermis of the blind side of pre-metamorphic larvae (Supplementary Fig. 39) increased adult chromatophores in postmetamorphic juveniles, whereas injection of retinol or DMSO had only a minor effect (Supplementary Figs. 40 and 41). In summary, we demonstrate that an RA gradient may be crucial for the generation of asymmetric pigmentation in post-metamorphic flatfish.

Recently, opsins in the skin of the adult octopus have been related to eye-independent color changes ${ }^{21}$. During flatfish metamorphosis there was ubiquitous expression in skin of genes of the phototransduction pathway (rh1, rh2, lws, sws1, and sws2) (Fig. 4b and Supplementary Figs. 42-46), although they were also in the skin of non-flatfish teleosts (Supplementary Figs. 47 and 48). Because opsins can regulate the synthesis of $\mathrm{RA}^{22}$, we hypothesized that the left-right RA gradient during asymmetric pigmentation and eye movement might result from increased light exposure on the ocular side due to the body tilt. This would then lead to an exclusive distribution of adult chromatophores on the ocular side at the latest stage of metamorphosis. To test this hypothesis, we exposed the blind and ocular sides of pre-metamorphic larvae to white light or light of different wavelengths. When both sides were exposed simultaneously to white light, the larval chromatophores distributed sporadically on both sides at $38 \mathrm{~d}$ after hatching (DAH) and were substantially increased in number in subsequent stages. Adult-type chromatophores started to proliferate at $50 \mathrm{DAH}$, achieving symmetrical pigmentation at $100 \mathrm{DAH}$ (Supplementary Fig. 49) ${ }^{23}$. Light of mid-length and long wavelengths (red, yellow, and green) caused a few chromatophore spots to appear on the blind side, whereas light of short wavelengths (blue and violet) caused a large number of chromatophores to appear (Fig. 4c, Supplementary Fig. 50, and Supplementary Table 32). Adult-type chromatophores developed evenly on both sides, resembling the situation after injection of RA. This suggests that light-sensing opsins under blue-light illumination may stimulate the production of RA. Most notably, the migration of the eye to the ocular side did not occur under blue-light treatment (Fig. 4c and Supplementary Fig. 50). The gene kit, which is related to pigment-cell development ${ }^{24}$, was co-expressed with the genes for blue-sensitive rho protein (sws2) and rxra in pigment cells (Fig. 4d). We did not observe co-expression with kit of $r h 1$ or opn4, at the wavelengths that overlap with sws 2 , or of other RA-related genes. This suggests that Sws2 promotes the synthesis of $\mathrm{RA}$, which in turn regulates Rxra during establishment of the left-right 
asymmetric pigmentation in flatfish. The involvement in this process of opsins in the eye remains an open question.

In summary, the establishment of a high-quality genome of the Japanese flounder and its comparison to the genome of another flatfish, the Chinese tongue sole, led to the identification of genes and developmental pathways that regulate metamorphosis and establish body asymmetry. Notably, the results uncover a role for the lightsensing opsins in establishing an RA gradient in the skin during metamorphosis. This functions as a developmental trigger after the dorso-ventral axis skew and augments asymmetric pigmentation. The identified cooperation between $\mathrm{TH}$ and RA signaling molecules in the regulation of eye migration and skin pigmentation is reminiscent of the situation in insect metamorphosis whereby ecdysteroids (Ecd) and juvenile hormone $(\mathrm{JH})$ have a critical joint role ${ }^{25}$. In common with THs, Ecd functions via a heterodimeric complex of two nuclear receptors, the ecdysone receptor and the $\mathrm{JH}$ receptor ultraspiracle (USP, the ortholog of Rxr) ${ }^{26}$, and 9-cis-RA is structurally similar to the terpenoid $\mathrm{JH}^{27,28}$. We propose that nuclear receptor transcription factors and terpenoids form the basis of the molecular mechanisms that trigger the unique metamorphosis found from invertebrates to vertebrates. Our detailed study of the molecular basis of flatfish metamorphosis reinforces the central role of endocrine factors in this late developmental event and a hitherto unexpected recapitulation of pivotal mechanisms regulating early development.

\section{METHODS}

Methods, including statements of data availability and any associated accession codes and references, are available in the online version of the paper.

Note: Any Supplementary Information and Source Data files are available in the online version of the paper.

\section{ACKNOWLEDGMENTS}

We sincerely thank Q.S. Tang, F.Z. Zhao, H.R. Lin and J.F. Gui for their support and help with the Japanese flounder genome project. We also thank A.V.M. Canário, J. Rotllant, Y.S. Fu, and J.A. Graves for constructive discussion. We acknowledge financial support from the National Natural Science Foundation of China (grants 31130057,31461163005 , and 31530078 to S.C.; grant 31472269 to C.S.; grant 31472262 to B.B.; and grant 31472273 to N.W.), the State 863 High Technology R\&D Project of China (grants 2012AA092203 and 2012AA10A408 to S.C.; grant 2012AA10A403-2 to C.S.), and the Education and Research of Guangdong Province (grant 2013B090800017 to Q.S.). This research also received funding from the Taishan Scholar Climb Project Fund of Shandong of China (S.C.), the Taishan Scholar Project Fund of Shandong of China for Young Scientists (C.S.), the Shanghai Universities First-class Disciplines Project of Fisheries (B.B.), the Program for Professor of Special Appointment (Eastern Scholar) at the Shanghai Institutions of Higher Learning (B.B.), and the Shanghai Municipal Science, Special Project on the Integration of Industry (B.B.).

\section{AUTHOR CONTRIBUTIONS}

S.C. and Q.W. initiated, managed, and drove the flounder genome sequencing project; S.C., M.S., Q.W., and D.M.P. conceived the study; C.S., B.B., S.C., D.M.P., and M.S. designed the analysis; S.C., B.B., Y.T., Y.L., and N.W. prepared the samples; Z.X., Q.Y., F.C., and J.Z. performed the genome assembly and annotation; C.S., W.L., X.J., Q.Y., B.L., X.L., L.W., Q.S., and S.M. performed the gene family and positive selection analysis; B.B., X.C., C.S., J.X., F.W., Z.D., J.G., W.C., W.X., Y.X., L.G., Z.S., and H.L. performed the experiments and data analysis; C.S., B.B., M.S., D.M.P., S.C., K.H., G.O., A.M.S., T.S., and G.Z. discussed the data. All authors contributed to data interpretation, and C.S., M.S., D.M.P., G.O., S.C., B.B., K.H., and A.M.S. wrote and revised the paper with significant contributions from all other authors.

\section{COMPETING FINANCIAL INTERESTS}

The authors declare no competing financial interests.

Reprints and permissions information is available online at http://www.nature.com/ reprints/index.html.
This work is licensed under a Creative Commons Attribution 4.0 Internationa (CC BY 4.0) license. The images or other third party material in this article is included in the article's Creative Commons license, unless otherwise indicated in the credit line; if the material is not included under the Creative Commons license, users will need to obtain permission from the license holder to reproduce the material. To view a copy of this license, visit http://creativecommons.org/licenses/by/4.0/

1. Friedman, M. The evolutionary origin of flatfish asymmetry. Nature 454, 209-212 (2008).

2. Laudet, V. The origins and evolution of vertebrate metamorphosis. Curr. Biol. 21, R726-R737 (2011).

3. Power, D.M. et al. The molecular and endocrine basis of flatfish metamorphosis Rev. Fish. Sci. 16, 95-111 (2008).

4. Schreiber, A.M. Flatfish: an asymmetric perspective on metamorphosis. Curr. Top. Dev. Biol. 103, 167-194 (2013).

5. Schreiber, A.M. Asymmetric craniofacial remodeling and lateralized behavior in larval flatfish. J. Exp. Biol. 209, 610-621 (2006).

6. Shao, C. et al. Genome-wide SNP identification for the construction of a highresolution genetic map of Japanese flounder (Paralichthys olivaceus): applications to QTL mapping of Vibrio anguillarum disease resistance and comparative genomic analysis. DNA Res. 22, 161-170 (2015)

7. Song, W. et al. Construction of high-density genetic linkage maps and mapping of growth-related quantitative trail loci in the Japanese flounder (Paralichthys olivaceus). PLoS One 7, e50404 (2012).

8. Chen, S. et al. Whole-genome sequence of a flatfish provides insights into ZW sex chromosome evolution and adaptation to a benthic lifestyle. Nat. Genet. 46, 253-260 (2014).

9. Inoue, T. et al. Involvement of the Hipk family in regulation of eyeball size, lens formation and retinal morphogenesis. FEBS Lett. 584, 3233-3238 (2010).

10. Li, L. et al. Change of eye shape during the metamorphosis in two flatfishes Paralichthys olivaceus and Solea senegalensis, with comparison of eye shape within the Pleuronectiformes. Ichthyol. Res. 60, 178-183 (2013).

11. Inui, Y. \& Miwa, S. Thyroid hormone induces metamorphosis of flounder larvae. Gen. Comp. Endocrinol. 60, 450-454 (1985).

12. Heimeier, R.A. \& Shi, Y.B. Amphibian metamorphosis as a model for studying endocrine disruption on vertebrate development: effect of bisphenol A on thyroid hormone action. Gen. Comp. Endocrinol. 168, 181-189 (2010).

13. St Germain, D.L., Galton, V.A. \& Hernandez, A. Minireview: defining the roles of the iodothyronine deiodinases: current concepts and challenges. Endocrinology 150, 1097-1107 (2009).

14. McMenamin, S.K. et al. Thyroid hormone-dependent adult pigment cell lineage and pattern in zebrafish. Science 345, 1358-1361 (2014).

15. Davis, P.J., Goglia, F. \& Leonard, J.L. Nongenomic actions of thyroid hormone. Nat. Rev. Endocrinol. 12, 111-121 (2016).

16. Campinho, M.A. et al. Flatfish metamorphosis: a hypothalamic independent process? Mol. Cell. Endocrinol. 404, 16-25 (2015).

17. Castillo, A.I. et al. A permissive retinoid $X$ receptor/thyroid hormone receptor heterodimer allows stimulation of prolactin gene transcription by thyroid hormone and 9-cis-retinoic acid. Mol. Cell. Biol. 24, 502-513 (2004).

18. Tanaka, Y., Okada, Y. \& Hirokawa, N. FGF-induced vesicular release of Sonic hedgehog and retinoic acid in leftward nodal flow is critical for left-right determination. Nature 435, 172-177 (2005).

19. Hamre, K. \& Harboe, T. Critical levels of essential fatty acids for normal pigmentation in Atlantic halibut (Hippoglossus hippoglossus L.) larvae. Aquaculture 277 101-108 (2008)

20. Hamre, K., Holen, E. \& Moren, M. Pigmentation and eye migration in Atlantic halibut (Hippoglossus hippoglossus L.) larvae: new findings and hypotheses. Aquacult. Nutr. 13, 65-80 (2007).

21. Ramirez, M.D. \& Oakley, T.H. Eye-independent, light-activated chromatophore expansion (LACE) and expression of phototransduction genes in the skin of Octopus bimaculoides. J. Exp. Biol. 218, 1513-1520 (2015).

22. McCaffery, P., Mey, J. \& Dräger, U.C. Light-mediated retinoic acid production. Proc. Natl. Acad. Sci. USA 93, 12570-12574 (1996).

23. Bolker, J.A. \& Hill, C.R. Pigmentation development in hatchery-reared flatfishes. J. Fish Biol. 56, 1029-1052 (2000).

24. Silver, D.L., Hou, L. \& Pavan, W.J. The genetic regulation of pigment cell development. Adv. Exp. Med. Biol. 589, 155-169 (2006).

25. Dhadialla, T.S., Carlson, G.R. \& Le, D.P. New insecticides with ecdysteroidal and juvenile hormone activity. Annu. Rev. Entomol. 43, 545-569 (1998).

26. Yao, T.P. et al. Functional ecdysone receptor is the product of EcR and UItraspiracle genes. Nature 366, 476-479 (1993).

27. Nakamura, A. et al. Effects of retinoids and juvenoids on moult and on phenoloxidase activity in the blood-sucking insect Rhodnius prolixus. Acta Trop. 103, 222-230 (2007).

28. Oro, A.E., McKeown, M. \& Evans, R.M. Relationship between the product of the Drosophila ultraspiracle locus and the vertebrate retinoid $\mathrm{X}$ receptor. Nature $\mathbf{3 4 7}$, 298-301 (1990).

29. Blomhoff, R. Vitamin $A$ in Health and Disease 1-35 (Marcel Dekker, Inc., New York, 1994).

30. Bao, B. et al. Proliferating cells in suborbital tissue drive eye migration in flatfish Dev. Biol. 351, 200-207 (2011). 


\section{ONLINE METHODS}

All samples used in this study were collected according to the guidelines for the care and use of experimental animals established by the Yellow Sea Fisheries Research Institute, CAFS. We estimated the number of samples necessary from the number of replicates required for sequencing and experiments. We did not use a randomized method and did not use a blinded methodology for group allocation during the experiments. We used Fisher's exact test, $t$-tests, one-way ANOVA and chi-square tests ${ }^{31}$ in the analysis of gene family evolution, positively selected genes, transcriptome data, $K$-mer distribution, GO and KEGG enrichment, RT-PCR, detection of RA concentration, and the effect of RA injection. When required, $P$ values were accordingly adjusted for multiple testing after considering the Benjamini-Hochberg false discovery rate (FDR) ${ }^{32}$. In the experiments involving immunohistochemistry and immunofluorescence, in situ RNA hybridization, in situ cell proliferation assay, irradiation treatment, yeast two-hybrid assay, and UPLC-MS/MS, we performed at least three independent or parallel experiments, and at least three individual larval specimens were treated in each group (at least three for positive and three for negative control) correspondingly.

Fish collection and maintenance. Gynogenetic Japanese flounder were temporarily maintained at the Key Laboratory of Sustainable Development of Marine Fisheries in Qingdao. The fish used for DNA extraction was anesthetized with MS-222 (Argent Chemical Laboratories, Redmond, WA, USA), and blood was collected and stored at $4{ }^{\circ} \mathrm{C}$ for later DNA isolation. The Japanese flounder and Chinese tongue sole larvae were produced from single batches of fertilized eggs that were maintained in single incubators at Huanghai Aquaculture Co. Ltd., Yantai, China. The larvae were fed live brine shrimp (Artemia nauplii) until the end of metamorphosis. The normal metamorphic stages were defined on the basis of eye migration: pre-metamorphosis (stage before eye migration; $16 \mathrm{~d}$ after hatching (DAH) in Japanese flounder, and $15 \mathrm{DAH}$ in tongue sole); pro-metamorphosis (eye begins to migrate; $21 \mathrm{DAH}$ in Japanese flounder, and $18 \mathrm{DAH}$ in tongue sole); metamorphic climax (upper edge of the migrating eye beyond the dorsal margin; $25 \mathrm{DAH}$ in Japanese flounder, and $19 \mathrm{DAH}$ in tongue sole), and post-metamorphosis (entire eye migrates past the dorsal midline; 29 DAH in Japanese flounder, and 22 DAH in tongue sole) ${ }^{33}$. We collected skin from the right (blind) and left (ocular) sides of Japanese flounder and tongue sole as asymmetrical pigmentation samples when the fish were about 3 months old. 2-mercapto-1-methylimidazole (0.3-0.34 g/l; Sigma-Aldrich, St. Louis, MO, USA) was dissolved directly in water and administered for $4 \mathrm{~d}$ to inhibit endogenous $\mathrm{TH}$ synthesis by metamorphic Japanese flounder. Larvae for whole-mount in situ RNA hybridization, immunohistochemistry, and cell proliferation analyses were anesthetized with $0.1 \mu \mathrm{g} / \mu \mathrm{l}$ MS-222, fixed in $4 \%$ paraformaldehyde (PFA) overnight, and stored in methanol at $4{ }^{\circ} \mathrm{C}$. Larvae for RNA and DNA isolation were preserved at $-80^{\circ} \mathrm{C}$.

Genome sequencing and assembly. We applied a whole-genome shotgun strategy and next-generation sequencing technology on an Illumina HiSeq 2000 platform to sequence the Japanese flounder genome. DNA was extracted from one gynogenetic female Japanese flounder according to the traditional phenol-chloroform extraction protocol ${ }^{8}$. To decrease the risk of nonrandomness, we constructed eight paired-end sequencing libraries with insert sizes of $170 \mathrm{bp}, 500 \mathrm{bp}, 800 \mathrm{bp}, 2 \mathrm{~kb}, 5 \mathrm{~kb}, 10 \mathrm{~kb}, 20 \mathrm{~kb}$, and $40 \mathrm{~kb}$ and sequenced them (ten lanes). The Japanese flounder genome was assembled via the de novo method using SOAPdenovo ${ }^{34}$. We used a $K$-mer-based method to estimate genome size, as described previously ${ }^{35}$.

Anchoring the scaffolds with genetic maps. We constructed high-resolution genetic maps for Japanese flounder using simple-sequence repeats and singlenucleotide polymorphisms, respectively ${ }^{6,7}$. The scaffolds, with markers located on different chromosomes, were filtered.

Whole-genome synteny analysis among different teleost species. Wholegenome alignments of Japanese flounder and tongue sole, Japanese flounder and zebrafish, and tongue sole and zebrafish were carried out on their repeat-masked genomes using LASTZ (version 1.02.00) $(T=2, \mathrm{C}=2$, $\mathrm{H}=2,000, \mathrm{Y}=3,400, \mathrm{~L}=5,500$, and $\mathrm{K}=2,000)$, and chainNetsoftware $\mathrm{c}^{36}$ was used to concatenate aligned fragments to longer fragments in a particular format, such as chainnet ${ }^{36}$.

Repeat annotation and comparison. We detected transposable elements (TEs) in the Japanese flounder genome by combining homology-based and $a b$ initio-based approaches. We used RepeatMasker (version open-3.2.9) against the RepBase TE library (RepBase 16.02) for the homology-based method and executed RepeatProteinMask. We constructed de novo repeat libraries by running PILER (version 1.0), RepeatScout (version 1.0.5), and LTR-Finder ${ }^{37}$ for the $a b$ initio-based approach and filtered the results to obtain nonredundant consensus repeat libraries. Finally, RepeatMasker was run on the genomes using these de novo libraries as reference libraries. Tandem repeats were detected in the genomic sequence data using Tandem Repeats Finder software ${ }^{38}$.

Transcriptome sequencing and analysis. Total RNA was isolated and purified from all of the samples by a TRIzol extraction method ${ }^{8}$. RNA concentration was measured using Nanodrop technology. RNAs of three samples were pooled in equal quantities to construct the Illumina sequencing libraries, and then paired-end sequences ( $90 \mathrm{bp}$ at each end) were generated using an Illumina HiSeq 2000.

The TopHat v1.2.0 package was used to map transcriptome reads to the genome and to identify DEGs. High-quality splice junctions were also predicted by TopHat. Gene expression was measured as reads per kilobase of gene per million mapped reads (RPKM) and adjusted using a scaling normalization method ${ }^{39}$. Only genes with RPKM $>1$ in at least one sequenced sample were considered. DEGs were detected using DEseq ${ }^{40}$ and Cuffdiff ${ }^{41}$. $P$ values were adjusted by the FDR ${ }^{32}$. Only genes with an adjusted $P<0.05$, obtained with any method, and fold change $>2$ were considered true DEGs. Genes were annotated to GO categories on the basis of the orthologous relationship between the Japanese flounder gene set and the tongue sole gene set, which had perfect GO annotation. Fisher's exact and chi-square tests were used to identify whether a list of genes (foreground genes) was enriched in a specific GO category as compared to the background genes. The KEGG automatic annotation server annotated the genes to KEGG pathways, using zebrafish and human as references ${ }^{42}$. The K-means algorithm was used to produce groups of DEGs using the calculated means mode with Euclidean distance based on the $\log$ fold-change data ${ }^{43}$.

Structural prediction and annotation of genes. We used a homology-based method, a de novo method, and an RNA-seq-based method to predict the genes in the Japanese flounder genome. Oreochromis niloticus, Cynoglossus semilaevis, Danio rerio, Gasterosteus aculeatus, Oryzias latipes, Takifugu rubripes, Tetraodon nigroviridis, and Homo sapiens proteins were used for the homology-based prediction, and the coding sequences were mapped onto the genome using TblastN $\left(1 \times 10^{-5}\right)$. Then, homologous genome sequences were aligned against matching proteins using Genewise to define the gene models ${ }^{44}$. Augustus (version 2.5.5) and Genscan (version 1.0) were used to predict coding genes de novo in the repeat-masked genome. The RNA-seq data were mapped to the genome using TopHat (version 1.2.0), and transcriptomebased gene structures were obtained with cufflinks (http://cufflinks.cbcb.umd. edu/). We did some manual checking to obtain the final reference gene set. We annotated the motifs and domains for the reference Japanese flounder gene set using InterPro against publicly available databases, including Pfam, ProDom, SMART, PRINTS, SUPERFAMILY, and PROSITE. The GO annotation information was abstracted with Iprscan (version 4.7). The Japanese flounder protein sequences were mapped to KEGG pathways to find the best hit for each gene. We also searched the Swiss-Prot and Trembl databases.

Gene family evolution. The coding and protein sequences from eight teleost species with sequenced genomes (O. niloticus, C. semilaevis, D. rerio, G. aculeatus, O. latipes, T. rubripes, T. nigroviridis, and P. olivaceus) and from human (as an outgroup species) were downloaded from the Ensemble genome site (release 60). We constructed a phylogenetic tree for the two flatfish and six other sequenced fish genomes ( $G$. aculeatus, O. latipes, T. rubripes, T. nigroviridis, O. niloticus, and $D$. rerio) using single-copy gene families. The coding sequences were extracted from each single-copy family and concatenated to 
one super-gene in the PHYLIP format for each species. Then, PhyML (v3.0) was used to construct the phylogenetic tree ${ }^{45}$. The Bayesian relaxed molecular clock (BRMC) approach ${ }^{46}$ was used to estimate species divergence time using the MCMCTREE program in the PAML package. CAFE-2.1 (ref. 47) was used to analyze changes in gene family size. A GO enrichment (MetaGO_ 20120303.RData object from GO.db package) analysis with a $P$ value $<0.05$ was performed on genes from the Japanese flounder expanding and contracting families.

Positive selection. All related genes of other species were downloaded from Ensembl release 64 for the positive selection analysis. MUSCLE was used for multiprotein sequence alignment among the flatfish genes and their orthologs using human proteins as reference. G blocks ${ }^{48}$ was used to eliminate poorly aligned positions and divergent regions in the DNA alignments, so they were suitable for the phylogenetic analysis. The filtered sequences were transformed to phylip format, and $\mathrm{PhyML}^{45}$ was used to reconstruct the phylogenetic tree with fourfold degenerate sites of singlecopy orthologous genes using the HKY 85 model. A branch model was used to detect the average $\omega$ across the tree $(\omega 0), \omega$ of the appointed branch to test $(\omega 2)$, and $\omega$ of all other branches $(\omega 1)$ using the following parameter settings: Codonfreq $=2, \kappa=2.5$, and initial $\omega=0.2$. Then, the chi-square test was used to check whether $\omega 2$ was significantly higher than $\omega 1$ and $\omega 0$, which would imply that the genes in the appointed branch had changed or evolved quickly. After obtaining positively selected genes $(\omega 2>\omega 1$ and $P<0.05)$, we performed a final manual check for genes of the phototransduction and the TH and RA signaling pathways.

Whole-mount immunohistochemistry and immunofluorescence. The negative controls of larvae used for immunohistochemistry were treated with a secondary antibody but not with the primary antibody. Whole-mount preparations were analyzed for triiodothyronine (T3) or thyroxine (T4) immunohistochemistry using horseradish peroxidase (HRP)-conjugated goat antimouse secondary antibody (Immunology Consultants Laboratory, Newberg, OR, USA) or fluorescein isothiocyanate (FITC)-conjugated goat anti-mouse secondary antibody (Fitzgerald) ${ }^{49}$ (Supplementary Note). Whole-mount specimens were observed and photographed under a dissecting microscope (Olympus, Tokyo, Japan) and documented using Image-Pro Plus6.0 software (Media Cybernetics, Bethesda, MD, USA). After the specimens had been rinsed in blocking solution (Roche), PBSTX (phosphate-buffered saline with Tween 20 and 5\% Triton X-100), and PBS, larval immunofluorescence was observed and photographed under an LSM710 NL0 laser scanning microscope (Carl Zeiss, Oberkochen, Germany).

Microinjection. We determined the effect of RA (Sigma-Aldrich) on eye migration in $16,18,21,23,26,28$, and $30 \mathrm{DAH}$ larvae by microinjecting $50 \mathrm{nl}$ into the suborbital area of the blind side: (i) no-injection control group 1 (natural development), (i) DMSO control group 2 (RA vehicle), (iii) all-trans RA group (ATRA, $2 \mathrm{mg} / \mathrm{ml}$ )), 9cRA group (9-cis-RA, $2 \mathrm{mg} / \mathrm{ml}$ ), and 13cRA group (13-cis-RA, $2 \mathrm{mg} / \mathrm{ml}$ ) (Supplementary Fig. 28). The larvae were maintained at $16-18{ }^{\circ} \mathrm{C}$ in $10-\mathrm{L}$ seawater containers kept in a controlled indoor rearing environment. The larvae ( $n=200$ per group) were microinjected using a Nikon IM-31 several days before putative initial eye migration. Several Japanese flounder larvae were treated with $0.1 \%$ 5-bromo-2'-deoxyuridine (BrdU) for an additional $8 \mathrm{~h}$ after RA injection to assess cell proliferation.

To study the effect of RA microinjection on asymmetrical body coloration in Japanese flounder, we divided the larvae into control (DMSO, RA vehicle), retinol, ATRA, and 9-cis-RA groups. The larvae were maintained at $16-18{ }^{\circ} \mathrm{C}$ in 10-L seawater containers kept in a controlled indoor rearing environment. The larvae ( $n=100$ per group) were microinjected at $28,31,34,37,40,43,47$ and 50 DAH with ATRA, 9-cis-RA, and retinol ( $2 \mathrm{mg} / \mathrm{ml}$ in DMSO) under the skin on the blind side (Supplementary Fig. 38).

Whole-mount in situ RNA hybridization. Total RNA was extracted from Japanese flounder larvae and reverse-transcribed using M-MLV reverse transcriptase (Promega, Madison, WI, USA) according to the manufacturer's instructions. The DNA was subjected to PCR using the primer combinations in Supplementary Table 33 and the following PCR reaction conditions: $95^{\circ} \mathrm{C}$ for $5 \mathrm{~min}$ followed by 40 cycles of $95^{\circ} \mathrm{C}$ for $1 \mathrm{~min}$, annealing temperature for $1 \mathrm{~min}, 72^{\circ} \mathrm{C}$ for $1 \mathrm{~min}$, and a final extension at $72^{\circ} \mathrm{C}$ for $5 \mathrm{~min}$. The PCR products were purified using a DNA gel extraction kit (Axygen, Tewksbury, MA, USA) and cloned into the pGEM-T Easy Vector system (Promega) according to the manufacturer's instructions. The validity and orientation of the cloned products within the plasmids were confirmed by sequencing. Both plasmids contained inserts in the same orientation in-frame with the T7 promoter and complementary to the SP6 promoter. Both sense and antisense RNA probes were prepared by in vitro transcription from the T7 and SP6 promoters, incorporating digoxigenin UTP (Roche). The plasmids were linearized with NotI or NcoI restriction enzymes to produce sense or antisense transcripts, respectively. The concentration of each labeled probe was determined by agarose gel electrophoresis, as described in the DIG RNA labeling kit (SP6/T7; Roche). The whole-mount in situ RNA hybridization was performed as previously described ${ }^{49}$ and is detailed in the Supplementary Note.

Two- and three-color fluorescence in situ RNA hybridization. The retinoid $\mathrm{x}$ receptor (rxrs) and retinoic acid receptor (rars) RNA probes were prepared for two-color FISH by in vitro transcription from the T7 and SP6 promoters, incorporating the Digoxigenin RNA labeling mix (Roche). We labeled related mRNAs with digoxigenin, biotin, and fluorescence for three-color FISH. $r x r a$, $r a r b, r h 1$, and opn4 were labeled with DIG RNA labeling mix (Roche), and sws 2 and kit were labeled with Biotin RNA labeling mix (Roche). sws2, kit, and $d c t$ were labeled with Fluorescence RNA labeling mix (Roche). Whole-mount in situ hybridization was carried out and processed using FISH methodology $y^{50}$ (Supplementary Note). The signal from the antisense probe was photographed using an LSM 710 confocal microscope (Carl Zeiss, Jena, Germany), whereas no signal was detected with the sense probe.

Whole-mount in situ cell proliferation assay. Larvae were treated with BrdU (Sigma-Aldrich) for $8 \mathrm{~h}$, anesthetized in $0.1 \mu \mathrm{g} / \mu \mathrm{l} \mathrm{MS}-222$, fixed in $4 \% \mathrm{PFA}$ overnight, and stored in methanol at $4{ }^{\circ} \mathrm{C}$. Larvae that were not labeled with $\mathrm{BrdU}$ were treated as negative controls. Whole-mount preparations were analyzed for BrdU immunostaining as previously described ${ }^{30}$ (Supplementary Note). Whole-mount specimens were observed and photographed using an SZX7 dissecting microscope (Olympus) and documented using Image-Pro Plus 6.0 software.

Irradiation treatment. Rectangular transparent plastic tanks $(35 \times 25 \times 15 \mathrm{~cm})$ were used to irradiate pre-metamorphic larvae. Light-emitting diodes (white, red, orange, yellow, green, blue, and violet) were set above and below the tanks to irradiate the larvae from two directions. We maintained the light intensity at the bottom of the tanks at 2,000 \pm 100 Lux by changing the distance between the lights and the tanks. Irradiation time was $12 \mathrm{~h} / \mathrm{d}$, and water temperature was $24 \pm 2{ }^{\circ} \mathrm{C}$. Three parallel experiments were set up for each light color, and each experiment included $16 \mathrm{DAH}$ individuals $(n=100)$. The irradiation treatment was carried out in an $80-\mathrm{m}^{2}$ dark room.

Exogenous THs rescue the nearly lethal effect of MMI treatment. Pre-metamorphic Japanese flounder larvae at $20 \mathrm{DAH}$, which had been reared in 10 liters of water at a constant temperature $\left(19^{\circ} \mathrm{C}\right)$, were separated into four groups (three treatment groups and one control group) containing 200 larvae per group. Another treatment group (group B), containing 800 larvae, was cultured in 30 liters of water under the same conditions. The larvae of the treatment groups were treated with $0.3 \mathrm{~g} / 1 \mathrm{MMI}$ for $4 \mathrm{~d}$, with one-third of the water replaced daily, and keeping the MMI concentration constant. Because of the high mortality of the Japanese flounder in the MMI treatment groups, we combined the three treatment groups containing 200 larvae into a single group (group A). Thereafter, the larvae were rescued by treatment with 10 p.p.b. T3 for $5 \mathrm{~d}$. In all the experiments the larvae were fed brine shrimp nauplii and rotifers.

Yeast two-hybrid assay. The coding regions of thraa and thrb1 of Japanese flounder were amplified using RT-PCR and ligated into pGBKT7 to generate pGBKT7-thraa and pGBKT7-thrb1. Similarly, the coding regions of rxra and $r x r b$ of Japanese flounder were amplified and ligated into pGADT7 to generate pGADT7-rxra and pGADT7- $r x r b$. In the self-activation assay, Y190 yeast cells 
were transformed with a single plasmid or a combination of plasmids. Y190 cells that were transformed with pGBKT7 were plated on plates containing synthetic dextrose (SD; Clontech) medium lacking tryptophan (SD - Trp) and were the negative control; Y190 cells transformed with pCL1, which encodes full-length wild-type GAL4 (galactose-responsive transcription factor, able to constitutively activate transcription), were plated on SD -Leu plates and were the positive control; Y190 cells transformed with pGBKT7-thraa and pGADT7 or with pGBKT7-thrb1 and pGADT7 were plated on SD -Leu-Trp plates; Y190 cells transformed with pGADT7-rxra and pGBKT7 or with pGADT7-rxrb and pGBKT7 were plated on SD -Leu-Trp plates. Transformed yeast cells were incubated at $30{ }^{\circ} \mathrm{C}$ for $72 \mathrm{~h}$. Colonies on filter paper were picked and transferred to a pool of liquid nitrogen for more than $30 \mathrm{~s}$. The papers were placed in a Z buffer-X-gal solution composed of $0.27 \mathrm{ml}$ of $\beta$-mercaptoethanol (SigmaAldrich), $1.67 \mathrm{ml}$ of X-gal stock solution ( $20 \mathrm{mg} / \mathrm{ml}$, Sigma-Aldrich), and $100 \mathrm{ml}$ of $\mathrm{Z}$ buffer, pH $7.0\left(\mathrm{Na}_{2} \mathrm{HPO}_{4} .7 \mathrm{H}_{2} \mathrm{O} 16.1 \mathrm{~g} / \mathrm{l}, \mathrm{NaH}_{2} \mathrm{PO}_{4} \cdot \mathrm{H}_{2} \mathrm{O} 5.5 \mathrm{~g} / \mathrm{l}\right.$, $\left.\mathrm{KCl} 0.75 \mathrm{~g} / \mathrm{l}, \mathrm{MgSO}_{4} \cdot \mathrm{H}_{2} \mathrm{O} 0.246 \mathrm{~g} / \mathrm{l}\right)$ in a clean $150-\mathrm{mm}$ plate and incubated at $30{ }^{\circ} \mathrm{C}$ until blue colonies appeared, which indicated self-activation of the plasmid. Yeast two-hybrid assays were performed according to the manufacturer's instructions. The two pairs of constructs (pGBKT7-thraa and pGADT7-rxra or pGBKT7-thrb1 and pGADT7-rxrb) were transformed into Y190 cells separately. pGBKT7-p53 and pGADT7-T antigen (positive control) or pGBKT7-lamin C and pGADT7-T antigen (negative control) were also transformed into Y190 cells. The cells were plated on SD -Leu-Trp plates. Positive colonies were identified as described above. All experiments were repeated at least three times.

Detection of RA concentration by ultraperformance liquid chromatography coupled to tandem mass spectrometry (UPLC-MS/MS). Skin was collected from the ocular and blind sides of post-metamorphic flounder juveniles (75 DAH). Skin $(0.1 \mathrm{~g})$ was ground in liquid nitrogen and transferred to glass centrifuge tubes, to which $10 \mu \mathrm{l}$ of $100 \mathrm{ng} / \mathrm{ml}$ acitretin (Aladdin, Shanghai, China) was added as an internal standard. Three volumes of $(\mu \mathrm{l} / \mathrm{mg}$, vol $/ \mathrm{wt})$ ice-cold $0.9 \% \mathrm{NaCl}$ and $1 \mathrm{ml}$ of $0.025 \mathrm{M} \mathrm{KOH}$ in ethanol were added to the extract. Then $5 \mathrm{ml}$ hexane was added before centrifugation for $5 \mathrm{~min}$ at 3,500 r.p.m. The hexane layer was removed, and the extraction procedure was repeated; then, $60 \mu \mathrm{l}$ of $4 \mathrm{M} \mathrm{HCl}$ was added to the aqueous phase, followed by $5 \mathrm{ml}$ of hexane before centrifugation as described above. The hexane phase, which contained the RA, was dried under a gentle stream of nitrogen, dissolved in $100 \mu \mathrm{l}$ of acetonitrile, and centrifuged at 12,000 r.p.m. for $3 \mathrm{~min}$. The supernatant was analyzed immediately by UPLC-MS/MS (see Supplementary Note for details).

Data availability. The sequencing reads have been deposited in the National Center for Biotechnology Information short-read archive for the Japanese flounder (P. olivaceus) genome (BioProject ID PRJNA73673) and the transcriptome sequences of Japanese flounder and tongue sole (C. semilaevis) (BioProject ID PRJNA296724).

31. Beissbarth, T. \& Speed, T.P. GOstat: find statistically overrepresented Gene Ontologies within a group of genes. Bioinformatics 20, 1464-1465 (2004).

32. Benjamini, $Y$. \& Hochberg, Y. Controlling the false discovery rate: a practical and powerful approach to multiple testing. J. R. Stat. Soc. Series B Stat. Methodol. 57, 289-300 (1995).

33. Takashi, M. The early life history of a flounder Paralichthys olivaceus. Nippon Suisan Gakk 48, 1581-1588 (1982)

34. Li, R. et al. De novo assembly of human genomes with massively parallel short read sequencing. Genome Res. 20, 265-272 (2010).

35. Li, R. et al. The sequence and de novo assembly of the giant panda genome. Nature 463, 311-317 (2010).

36. Kent, W.J., Baertsch, R., Hinrichs, A., Miller, W. \& Haussler, D. Evolution's cauldron duplication, deletion, and rearrangement in the mouse and human genomes. Proc. Natl. Acad. Sci. USA 100, 11484-11489 (2003).

37. Xu, Z. \& Wang, H. LTR_FINDER: an efficient tool for the prediction of full-length LTR retrotransposons. Nucleic Acids Res. 35, W265-W268 (2007).

38. Benson, G. Tandem repeats finder: a program to analyze DNA sequences. Nucleic Acids Res. 27, 573-580 (1999).

39. Robinson, M.D. \& Oshlack, A. A scaling normalization method for differential expression analysis of RNA-seq data. Genome Biol. 11, R25 (2010).

40. Anders, S. \& Huber, W. Differential expression analysis for sequence count data. Genome Biol. 11, R106 (2010).

41. Roberts, A., Trapnell, C., Donaghey, J., Rinn, J.L. \& Pachter, L. Improving RNA-Seq expression estimates by correcting for fragment bias. Genome Biol. 12, R22 (2011).

42. Moriya, Y., Itoh, M., Okuda, S., Yoshizawa, A.C. \& Kanehisa, M. KAAS: an automatic genome annotation and pathway reconstruction server. Nucleic Acids Res. 35 W182-W185 (2007)

43. Eisen, M.B., Spellman, P.T., Brown, P.O. \& Botstein, D. Cluster analysis and display of genome-wide expression patterns. Proc. Natl. Acad. Sci. USA 95, 14863-14868 (1998).

44. Birney, E., Clamp, M. \& Durbin, R. GeneWise and Genomewise. Genome Res. 14 988-995 (2004).

45. Guindon, S. et al. New algorithms and methods to estimate maximum-likelihood phylogenies: assessing the performance of PhyML 3.0. Syst. Biol. 59, 307-321 (2010).

46. Kishino, H., Thorne, J.L. \& Bruno, W.J. Performance of a divergence time estimation method under a probabilistic model of rate evolution. Mol. Biol. Evol. 18, 352-361 (2001).

47. De Bie, T., Cristianini, N., Demuth, J.P. \& Hahn, M.W. CAFE: a computational tool for the study of gene family evolution. Bioinformatics 22, 1269-1271 (2006).

48. Talavera, G. \& Castresana, J. Improvement of phylogenies after removing divergent and ambiguously aligned blocks from protein sequence alignments. Syst. Biol. $\mathbf{5 6}$, 564-577 (2007)

49. Bao, B. et al. Isolation of SFRS3 gene and its differential expression during metamorphosis involving eye migration of Japanese flounder Paralichthys olivaceus. Biochim. Biophys. Acta 1725, 64-70 (2005).

50. Jeong, J.K., Chen, Z., Tremere, L.A. \& Pinaud, R. Double fluorescence in situ hybridization in fresh brain sections. J. Vis. Exp. 42, 2102 (2010). 